\title{
Электрохимические исследования процессов легирования медью слоистых кристаллов семейства $[(\mathrm{Ge}, \mathrm{Sn}, \mathrm{Pb})(\mathrm{Te}, \mathrm{Se})]_{m}\left[(\mathrm{Bi}, \mathrm{Sb})_{2}(\mathrm{Te}, \mathrm{Se})_{3}\right]_{n}(m, n=0,1,2 \ldots)$
}

\author{
(C) М.А. Кретова, М.А. Коржуев , Е.С. Авилов
}

Институт металлургии и материаловедения им. А.А.Байкова Российской академии наук, 119334 Москва, Россия

I E-mail: korzhuev@imet.ac.ru

(Получена 27 декабря 2016 г. Принята к печати 12 января 2017 г.)

Изучены процессы интеркаляции меди в ван-дер-ваальсовы (VdW) щели слоистых тройных сплавов семейства $[(\mathrm{Ge}, \mathrm{Sn}, \mathrm{Pb})(\mathrm{Te}, \mathrm{Se})]_{m}\left[(\mathrm{Bi}, \mathrm{Sb})_{2}(\mathrm{Te}, \mathrm{Se})_{3}\right]_{n}(m, n=0,1,2 \ldots)$, изменяющие электрические, механические и другие физические свойства образцов. Обнаружено пропорциональное снижение концентрации

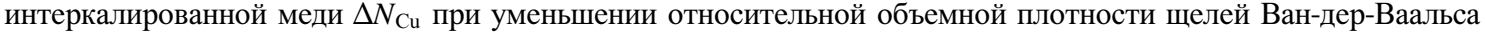
$D_{\mathrm{VdW}}=1 / s$ с ростом слойности пакетов $s$, а также их толщины $\xi_{1}$ при изменении состава тройных сплавов.

DOI: 10.21883/FTP.2017.07.44647.33

\section{1. Введение}

Слоистые кристаллы $(\mathrm{CK})$ типа $\mathrm{Bi}_{2} \mathrm{Te}_{3}$ (структурный тип тетрадимита $\mathrm{Bi}_{2} \mathrm{Te}_{2} \mathrm{~S}$, пространственная группа симметрии $R \overline{3} m)$ состоят из 5-слойных пакетов $(s 5)$, разделенных щелями Ван-дер-Ваальса (VdW) [1]. Химическая связь в щелях VdW существенно ослаблена, поэтому в них легко могут быть интеркалированы быстро диффундирующие примеси (доноры $\mathrm{Cu}, \mathrm{Ag}$ и др.), изменяющие электрические и другие физические свойства сплавов [2,3]. Ранее на основе бинарных сплавов (БС) типа $\mathrm{Bi}_{2} \mathrm{Te}_{3}$ были синтезированы тройные сплавы (TC) семейства $[(\mathrm{Ge}, \mathrm{Sn}, \mathrm{Pb})(\mathrm{Te}, \mathrm{Se})]_{m}\left[(\mathrm{Bi}, \mathrm{Sb})_{2}(\mathrm{Te}, \mathrm{Se})_{3}\right]_{n}$ $(m, n=0,1,2 \ldots)$ с измененной по сравнению с $\mathrm{Bi}_{2} \mathrm{Te}_{3}$ слойностью [4,5]. Полученные ТС имеют более сложную структуру слоев (пакеты $s 5, s 7, s 9, s 11, s 13 \ldots$ ). При этом в зависимости от соотношения $m / n$ толщина слоевых пакетов в них меняется в пределах $\xi_{1}=1-3 \mathrm{Hм}$, а период сверхструктуры вдоль тригональной оси $\overline{3}-$ в пределах $\xi_{2}=2-18$ нм [6]. Соответствующее изменение

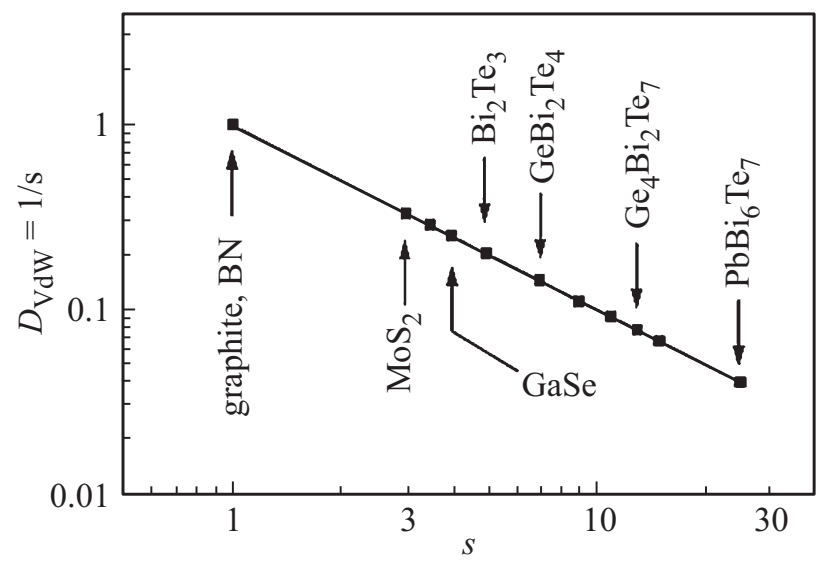

Рис. 1. Сравнение относительной объемной плотности щелей Ван-дер-Ваальса $D_{\mathrm{Vdw}}=1 / s$ в зависимости от слойности пакетов $s$ в различных СК [7]. относительной плотности щелей $\mathrm{VdW} \quad D_{\mathrm{VdW}}=1 / s$ в зависимости от слойности $s$ пакетов в кристаллах типа $\mathrm{Bi}_{2} \mathrm{Te}_{3}$ в сравнении с другими СК показано на рис. 1 [7]. Из рис. 1 видно, что в кристаллах типа $\mathrm{Bi}_{2} \mathrm{Te}_{3}$ при переходе БС $\rightarrow$ ТC величина $D_{\mathrm{VdW}}$ уменьшается. Можно предположить, что уменьшение $D_{\mathrm{VdW}}$ может приводить к изменению равновесной концентрации примесей, которые можно интеркалировать в щели VdW семейства TC $[(\mathrm{Ge}, \mathrm{Sn}, \mathrm{Pb})(\mathrm{Te}, \mathrm{Se})]_{m}\left[(\mathrm{Bi}, \mathrm{Sb})_{2}(\mathrm{Te}, \mathrm{Se})_{3}\right]_{n}$ $(m, n=0,1,2 \ldots)$.

Целью настоящей работы было экспериментальное исследование процессов интеркаляции меди в ТС различных составов методом автоэлектрохимического легирования (АЭХЛ) образцов [2,3].

\section{2. Эксперимент}

Для исследований использовали поликристаллические сплавы: I - $(\mathrm{GeTe})_{m}\left(\mathrm{Bi}_{2} \mathrm{Te}_{3}\right)_{n} ; \mathrm{II}-(\mathrm{GeTe})_{m}\left(\mathrm{Sb}_{2} \mathrm{Te}_{3}\right)_{n}$ и III - $(\mathrm{PbTe})_{m}\left(\mathrm{Bi}_{2} \mathrm{Te}_{3}\right)_{n}$. Сплавы синтезировали при температуре $T=1020-1190 \mathrm{~K}$, а затем охлаждали со скоростью $4 \mathrm{~K} /$ мин до $T=770 \mathrm{~K}$. При этой температуре сплавы отжигали в течение 200-400 ч, а затем закаливали погружением в воду. Использовали образцы $(\mathrm{X})$ произвольной формы (массой $m \approx 3-4$ г), которые помещали в электрохимическую ячейку (ЭХЯ) $\left\langle\mathrm{X} /\right.$ aq. $\left.\mathrm{CuSO}_{4} / \mathrm{Cu}\right\rangle$ [2,3]. АЭХЛ проводили в течение $t=100$ ч в короткозамкнутых ЭХЯ путем помещения образца с надетым на него медным „хомутом“ непосредственно в электролит [2].

До/после АЭХЛ измеряли эдс разомкнутых ЭХЯ:

$$
E=-\left(\mu_{\mathrm{Cu}}^{\mathrm{X}}-\mu_{\mathrm{Cu}}^{0}\right) / Z e .
$$

Здесь $\mu_{\mathrm{Cu}}^{\mathrm{X}}$ и $\mu_{\mathrm{Cu}}^{0}-$ электрохимические потенциалы атомов меди в исследуемых образцах $(\mathrm{X})$ и в металлической меди, $Z e$ - заряд, переносимый ионом меди $\mathrm{Cu}^{2+}$ в электролите $(Z=2), e-$ элементарный заряд. 
Слойность образцов $\bar{s}$, эдс $E$ ЭХЯ, термоэдс $\alpha$, а также концентрация меди $\Delta N_{\mathrm{Cu}}$, интеркалированной в образцы $(T=300 \mathrm{~K})$

\begin{tabular}{|c|c|c|c|c|c|c|c|}
\hline $\begin{array}{c}\text { Номер } \\
\text { образца }\end{array}$ & $\begin{array}{c}\text { Состав и тип } \\
\text { проводимости } \\
\text { образцов }\end{array}$ & $\bar{s}$ & $\begin{array}{l}E_{0}, \\
\mathrm{MB}\end{array}$ & $\begin{array}{c}E, \\
\mathrm{MB}\end{array}$ & $\begin{array}{c}\alpha_{0}, \\
\mathrm{M \kappa B} / \mathrm{K}\end{array}$ & $\begin{array}{c}\alpha, \\
\text { мкB/K }\end{array}$ & $\begin{array}{c}\Delta N_{\mathrm{Cu}}, \\
10^{19} \mathrm{~cm}^{-3}\end{array}$ \\
\hline 1 & $p-\operatorname{GeTe}(\alpha)$ & 2 & 90.4 & 75.6 & 30 & 36 & 0.5 \\
\hline 2 & $p-\mathrm{Ge}_{9} \mathrm{Bi}_{2} \mathrm{Te}_{12}$ & 7.7 & 110.2 & 62.4 & 87 & 67 & - \\
\hline 3 & $p-\mathrm{Ge}_{8} \mathrm{Bi}_{2} \mathrm{Te}_{11}$ & 7 & -19.2 & 61.1 & 88 & 58 & - \\
\hline 4 & $p-\mathrm{Ge}_{7} \mathrm{Bi}_{2} \mathrm{Te}_{10}$ & 6.3 & -44.4 & 20.3 & 87 & 54 & - \\
\hline 5 & $p-\mathrm{Ge}_{6} \mathrm{Bi}_{2} \mathrm{Te}_{9}$ & 5.7 & 80.9 & 33.8 & 102 & 58 & - \\
\hline 6 & $p-\mathrm{Ge}_{5} \mathrm{Bi}_{2} \mathrm{Te}_{8}$ & 15 & 24.2 & 42.7 & 102 & 59 & - \\
\hline 7 & $p-\mathrm{Ge}_{4} \mathrm{Bi}_{2} \mathrm{Te}_{7}$ & 13 & -30.2 & -25.8 & 133 & 61 & - \\
\hline 8 & $p-\mathrm{Ge}_{3} \mathrm{Bi}_{2} \mathrm{Te}_{6}$ & 11 & -20.4 & 81.6 & 129 & 62 & - \\
\hline 9 & $p-\mathrm{Ge}_{2} \mathrm{Bi}_{2} \mathrm{Te}_{5}$ & 9 & 154.6 & 50.8 & 93 & 43 & - \\
\hline 10 & $n-\mathrm{Ge}_{1,2} \mathrm{Bi}_{2} \mathrm{Te}_{4}$ & 7 & 61.8 & -82.1 & -104 & -75 & 2 \\
\hline 11 & $n-\mathrm{GeBi}_{2} \mathrm{Te}_{4}$ & 7 & 150.3 & 140.1 & -81 & -62 & 2 \\
\hline 12 & $n-\mathrm{GeBi}_{4} \mathrm{Te}_{7}$ & 6 & 129.7 & 45 & -110 & -65 & 3.5 \\
\hline 14 & $n-\mathrm{GeBi}_{6} \mathrm{Te}_{10}$ & 5.5 & 136.8 & 36.1 & 13 & -69 & 3 \\
\hline 15 & $n-\mathrm{GeBi}_{8} \mathrm{Te}_{13}$ & 5.4 & 130.1 & 39.7 & -75 & -75 & 0 \\
\hline 16 & $n-\mathrm{GeBi}_{10} \mathrm{Te}_{16}$ & 5.8 & 135.9 & 38.2 & -89 & -33 & 3 \\
\hline 17 & $p-\mathrm{Bi}_{2} \mathrm{Te}_{3}$ & 5 & 135.8 & 23.3 & 341 & -161 & 4 \\
\hline 18 & $p-\mathrm{Ge}_{5} \mathrm{Sb}_{2} \mathrm{Te}_{8}$ & 13 & 132.1 & 8.95 & 28 & 33 & 1 \\
\hline 19 & $p-\mathrm{Ge}_{4} \mathrm{Sb}_{2} \mathrm{Te}_{7}$ & 15 & 105 & 40.5 & 34 & 34 & 0 \\
\hline 20 & $p-\mathrm{Ge}_{3} \mathrm{Sb}_{2} \mathrm{Te}_{6}$ & 11 & 146.1 & -30.3 & 29 & 32 & 2 \\
\hline 21 & $p-\mathrm{Ge}_{2} \mathrm{Sb}_{2} \mathrm{Te}_{5}$ & 9 & 147.9 & -43.7 & 28 & 37 & 3 \\
\hline 22 & $p-\mathrm{GeSb}_{2} \mathrm{Te}_{4}$ & 7 & 133 & 10.9 & 29 & 39 & 4 \\
\hline 23 & $p-\mathrm{GeSb}_{4} \mathrm{Te}_{7}$ & 6 & 125.1 & 17.2 & 30 & 38 & 5 \\
\hline 24 & $p-\mathrm{GeSb}_{6} \mathrm{Te}_{10}$ & 5.7 & 161.5 & 129.6 & 28 & 49 & 4.5 \\
\hline 25 & $p-\mathrm{GeSb}_{8} \mathrm{Te}_{13}$ & 5.5 & 143.1 & 20.5 & 28 & 30 & 3 \\
\hline 32 & $p$-PbTe & 2 & 43.2 & 26.7 & -230 & -151 & 2 \\
\hline 35 & $n-\mathrm{PbBi}_{2} \mathrm{Te}_{4}$ & 7 & 145 & 9.1 & -56 & -47 & 2 \\
\hline 36 & $n-\mathrm{Pb}_{2} \mathrm{Bi}_{6} \mathrm{Te}_{11}$ & 6.3 & 132.5 & 47.4 & -40 & -37 & 1 \\
\hline 37 & $n-\mathrm{PbBi}_{4} \mathrm{Te}_{7}$ & 6 & 138.8 & -7.6 & -56 & -49 & 2 \\
\hline 38 & $n-\mathrm{PbBi}_{6} \mathrm{Te}_{10}$ & 5.7 & 110.3 & 92.3 & -41 & -40 & 0 \\
\hline 39 & $n-\mathrm{PbBi}_{8} \mathrm{Te}_{13}$ & 5.5 & 78.95 & 40.4 & -61 & -53 & 1 \\
\hline
\end{tabular}

Примечание. Индексом (0) отмечены значения $E$ и $\alpha$ до АЭХЛ.

Для измерений $E$ использовали вольтметр Щ300 с входным сопротивлением $R_{\text {in }}>1000$ МОм. По величине и знаку $E$ определяли возможность интеркаляции меди в сплавы („+““ на образце - да; „-“ — нет), а также степень близости результатов АЭХЛ к равновесным условиям $(E=0)$. Количество меди $\Delta N_{\mathrm{Cu}}$, введенной в образцы при АЭХЛ, определяли методом измерения термоэдс $\alpha$ образцов до/после АЭХЛ [1,2]. ${ }^{1}$

Всего было исследовано 39 образцов различного состава (см. таблицу).

\section{3. Экспериментальные результаты}

В таблице и на рис. 2 показаны результаты измерения эдс $E$ ЭХЯ, а также концентрации меди $\Delta N_{\mathrm{Cu}}$, интеркалированной в образцы, в зависимости от состава, от $n /(n+m)$ ТС, а также параметров $\xi_{1}\left(\bar{\xi}_{1}\right)$ и $s(\bar{s})$. Здесь $\bar{\xi}_{1}$ и $\bar{s}-$ средние взвешенные значения $\xi_{1}$ и $s$ для

\footnotetext{
1 Для образцов 2-9 (см. таблицу), находящихся вблизи перехода $p \rightarrow n$, величину $\Delta N_{\mathrm{Cu}}$ по изменениям $\alpha$ точно оценить не удалось.
}

мультипакетных ТС. Штриховыми линиями на рис. 1 и 2 показаны соответствующие характеристики сплавов $\mathrm{Bi}_{2} \mathrm{Te}_{3}$, пунктиром - общие статистические линейные тренды исследуемых зависимостей.

Согласно рис. 2, $a$, величина $E$, измеренная до АЭХЛ, возрастала с ростом $n /(n+m)$ (пунктирная линия). В свою очередь, концентрация меди $\Delta N_{\mathrm{Cu}}$, интеркалированной в образцы, также возрастала с ростом величины $E$ (рис. $2, b)$, и убывала с ростом $\xi_{1}\left(\bar{\xi}_{1}\right)$ и $s(\bar{s})$ (пунктирная линия на рис. $1, c$ ). При переходе от $\mathrm{Bi}_{2} \mathrm{Te}_{3}$ к ТС величина $\Delta N_{\mathrm{Cu}}$ понижалась, что соответствует увеличению слойности $s$ и снижению относительной плотности щелей $\mathrm{VdW} D_{\mathrm{VdW}}=1 / s$ (рис. 1 и 2). С ростом параметров $s$ и $\bar{\xi}_{1}$ величины $E$ и $\Delta N_{\mathrm{Cu}} \sim 1 / s \sim 1 / \bar{\xi}_{1}$ убывали в пропорции, близкой к 1:1 (пунктирная линия на рис. $2, c)$. Снижение $E$ и $\Delta N_{\mathrm{Cu}}$ мы объясняем уменьшением объемной плотности щелей Ван-дер-Ваальса $D_{\mathrm{VdW}}=1 / s$ в образцах с ростом $s$ и $\xi_{1}$ (рис. 1$)$.

После проведения АЭХЛ для большинства образцов выполнялось условие $E \rightarrow 0$, что соответствует предельной равновесной концентрации меди $\Delta N_{\mathrm{Cu}}$, интер- 

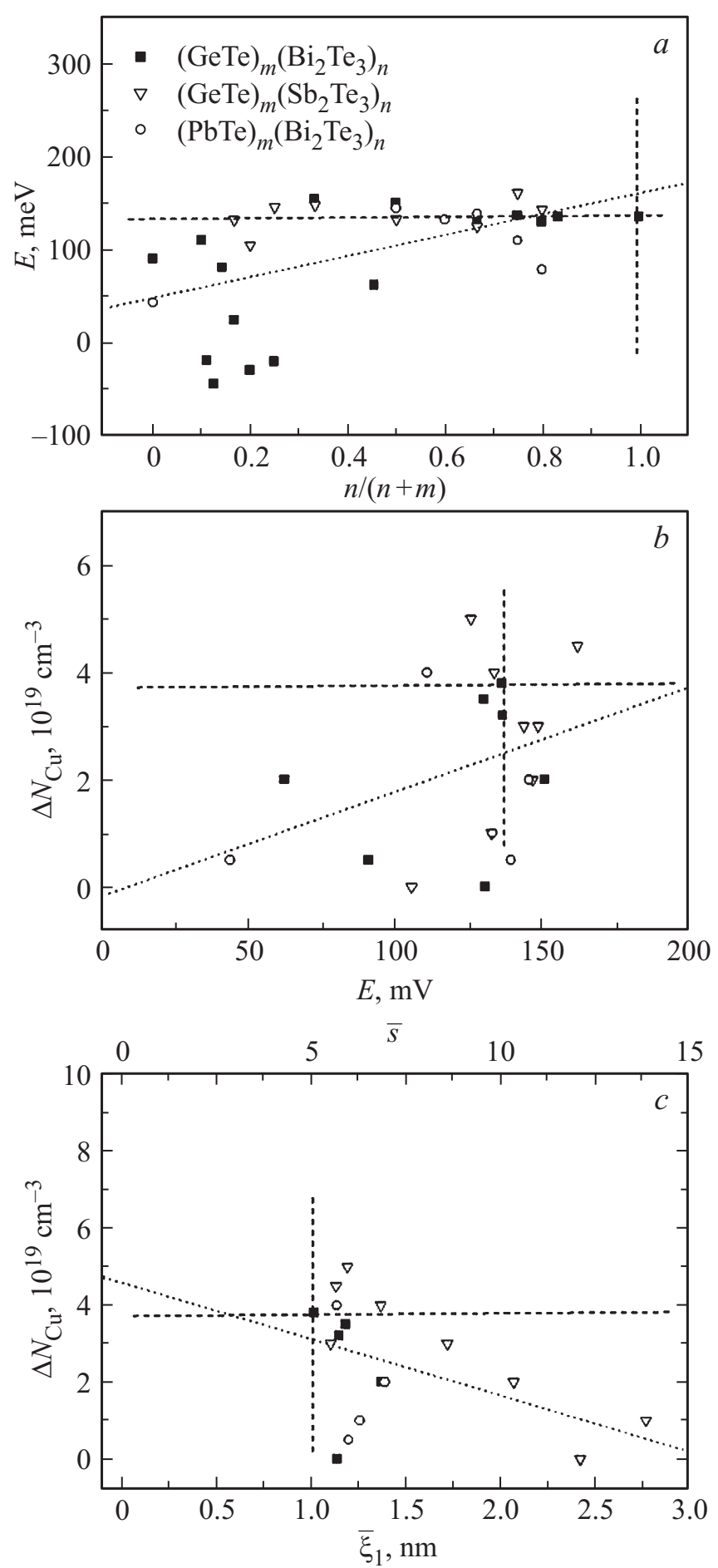

Рис. 2. Зависимости эдс $E$ разомкнутых ЭХЯ от состава $(a)$, концентрации меди $\Delta N_{\mathrm{Cu}}$, интеркалированной в образцы, от эдс $E$ ЭХЯ (b) и параметров $\bar{s}, \bar{\xi}_{1}$ ТC $(c)$.

калированной в образцы (см. таблицу). Соотношения $E>0$, наблюдаемые для отдельных образцов после АЭХЛ (см. таблицу), можно объяснить их недостаточно равновесным состоянием, связанным с внутренними механическими напряжениями, обычно присутствующими в поликристаллах.

\section{4. Заключение}

Таким образом, обнаружено пропорциональное снижение концентрации меди $\Delta N_{\mathrm{Cu}}$, интеркалированной в слоистые образцы $[(\mathrm{Ge}, \mathrm{Sn}, \mathrm{Pb})(\mathrm{Te}, \mathrm{Se})]_{m}\left[(\mathrm{Bi}, \mathrm{Sb})_{2}(\mathrm{Te}, \mathrm{Se})_{3}\right]_{n}$ $(m, n=0,1,2 \ldots)$, с ростом слойности пакетов $s(\bar{s})$, a также их средней толцины $\xi_{1}\left(\bar{\xi}_{1}\right)$, связанное с уменьшением относительной объемной плотности щелей Ван-дер-Ваальса $D_{\mathrm{VdW}}=1 / s$ в образцах.

\section{Список литературы}

[1] Б.М. Гольцман, В.А. Кудинов, И.А. Смирнов. Полупроводниковые термоэлектрические материалы на основе $\mathrm{Bi}_{2} \mathrm{Te}_{3}$ (М., Наука, 1972) с. 14.

[2] М.А. Коржуев, Т.Е. Свечникова. ФТП, 25 (12), 2141 (1991).

[3] М.А. Коржуев. ФТТ, 38 (3), 883 (1996).

[4] Л.Е. Шелимова, О.Г. Карпинский, П.П. Константинов, Е.С. Авилов, М.А. Кретова, В.С. Земсков. Неорг. матер., 37 (4), 421 (2001); Неорг. матер., 40 (5), 451 (2004).

[5] В.С. Земсков, Л.Е. Шелимова, О.Г. Карпинский, П.П. Константинов, Е.С. Авилов, М.А. Кретова, И.Ю. Нихезина. Термоэлектричество, № 1, 18 (2010); Термоэлектричество, № 1,18 (2012).

[6] М.А. Коржуев, И.В. Катин, М.А. Кретова, Е.С. Авилов. Механические свойства современных конструкционных материалов (М., ИМЕТ РАН, 2016) с. 65.

[7] М.А. Кретова, М.А. Коржуев, А.Б. Михайлова, И.В. Катин, Е.С. Авилов. Механические свойства современных конструкционных материалов (М., ИМЕТ РАН, 2016) с. 73.

Редактор Л.В. Шаронова

\section{Electrochemical studies of layered crystals family $[(\mathrm{Ge}, \mathrm{Sn}, \mathrm{Pb})(\mathrm{Te}, \mathrm{Se})]_{m}\left[(\mathrm{Bi}, \mathrm{Sb})_{2}(\mathrm{Te}, \mathrm{Se})_{3}\right]_{n}$ $(m, n=0,1,2 \ldots)$ doped with copper}

\section{M.A. Kretova, M.A. Korzhuev, E.S. Avilov}

Baikov Institute of Metallurgy and Material Science, Russian Academy of Sciences, 119334 Moscow, Russia

Abstract The processes of intercalation of copper in the Van der Waals (VdW) slit of layered ternary alloys of the family $[(\mathrm{Ge}, \mathrm{Sn}, \mathrm{Pb})(\mathrm{Te}, \mathrm{Se})]_{m}\left[(\mathrm{Bi}, \mathrm{Sb})_{2}(\mathrm{Te}, \mathrm{Se})_{3}\right]_{n} \quad(m, n=0,1,2 \ldots)$, which change the electrical, mechanical and other physical properties of the samples, are studied. A proportional reduction in intercalated copper concentration $\Delta N_{\mathrm{Cu}}$ is detected with decreasing relative bulk density of Van der Waals slits $D_{\mathrm{VdW}}=1 / s$, and with increasing package ply $s$, and their thickness $\xi_{1}$ in the samples at composition changing. 